\title{
Laboratorios virtuales en entorno de aprendizaje de química orgánica, para el bachillerato ecuatoriano
}

\author{
Fecha de recepción : 2021-05-03 • Fecha de aceptación: 2021-07-17 • Fecha de publicación: 2021-09-10
}

\author{
María Fernanda Arroba Arroba ${ }^{1}$ \\ Pontificia Universidad Católica de Ecuador, Ecuador \\ maria.f.arroba.a@pucesa.edu.ec \\ https://orcid.org/0000-0002-8216-3959 \\ Santiago Alejandro Acurio Maldonado² \\ Pontificia Universidad Católica de Ecuador, Ecuador \\ sacurio@pucesa.edu.ec \\ https://orcid.org/0000-0001-7069-9206
}

\section{Resumen}

La importancia de este estudio sobresale en la implementación de laboratorios virtuales con los estudiantes de tercero de bachillerato de la Unidad Educativa Vicente Anda Aguirre (Ecuador), herramienta que beneficia en la adquisición de saberes que mediante el aprovechamiento de los avances tecnológicos promueve la realización de actividades prácticas, que contribuyen en el refuerzo del conocimiento; lamentablemente, la insuficiente asimilación de los conceptos de química orgánica se refleja en la falta de entusiasmo, y el desinterés para enfrentar retos académicos con autonomía, factor que perjudica en la experiencia del alumno, la participación, experimentación, el análisis y la toma de decisiones; es importante resaltar que por la emergencia sanitaria COVID-19 que afecta a la población, el estudiante no realiza las prácticas en un laboratorio real. 
Por las razones expuestas, el objetivo del estudio es implementar estrategias didácticas en entornos virtuales en el proceso de aprendizaje de los contenidos de química orgánica. La indagación se enmarca en un enfoque cuantitativo, la metodología se enfoca en el aprendizaje basado en problemas (ABP), con un alcance correlacional, descriptivo y explicativo. En el proceso de implementación de los laboratorios virtuales, se analiza la capacidad del estudiantado para construir y comprender argumentos, representaciones o modelos que incluyen experimentos llevados a efecto en la virtualidad. Desde este concepto se diseñan estrategias aplicables en el aula de acuerdo a la propuesta curricular y al método de evaluación.

\section{Palabras clave: aprendizaje, entorno virtual, estrategias didácticas, entorno virtual, química} orgánica.

\section{Abstract}

The importance of this study stands out in the implementation of virtual laboratories with third year high school students of the Vicente Anda Aguirre Educational Unit (Ecuador), a tool that benefits in the acquisition of knowledge that through the use of technological advances promotes the realization of practical activities, which contribute to the reinforcement of knowledge; Unfortunately, the insufficient assimilation of the concepts of organic chemistry is reflected in the lack of enthusiasm and disinterest to face academic challenges with autonomy, a factor that harms the student's experience, participation, experimentation, analysis and decision making; it is important to highlight that due to the COVID-19 sanitary emergency that affects the population, the student does not perform the practices in a real laboratory.

For the above reasons, the objective of the study is to implement didactic strategies in virtual environments in the learning process of organic chemistry contents. The inquiry is framed in a quantitative approach, the methodology focuses on problem-based learning (PBL), with a correlational, descriptive and explanatory scope. In the process of implementing virtual laboratories, the students' ability to construct and understand arguments, representations or models that include experiments carried out virtually is analyzed. From this concept, strategies applicable in the classroom are designed according to the curricular proposal and the evaluation method.

Keywords: didactic strategies, virtual environment, organic chemistry, learning. 


\section{Introducción}

En el bachillerato general unificado, la asignatura de química orgánica es considerada una materia difícil en su comprensión, el entendimiento y el aprendizaje, con un alto índice de reprobación. En el estudio realizado por Bermeo et al. (2018), analizan las causas que afectan en el aprendizaje de la asignatura a los estudiantes de la Facultad de Ciencias Químicas de la Universidad Central del Ecuador, que alcanza el $62,4 \%$ de la tasa de repetición en los primeros semestres; entre los principales causales se hace referencia al bajo nivel de conocimientos de los educandos del nivel educativo secundario, la inadecuada aplicación de recursos tecnológicos, la insuficiente planeación de las actividades a realizar y la práctica pedagógica tradicional, los laboratorios convencionales.

En la investigación realizada por Sepúlveda (2014), identifica una diversidad de trabajos relacionados con la química orgánica, evidenciando que los estudiantes de secundaria exteriorizan dificultades durante el aprendizaje: en la formulación y nomenclatura de compuestos orgánicos, reacciones y ecuaciones químicas, balanceo de ecuaciones químicas, estequiometria de ecuaciones químicas, las soluciones, los gases, cinética y equilibrio químico. Por lo expuesto, es trascendental la utilización de la tecnología; herramienta utilizada para facilitar los procesos de adquisición del saber que facilitan nuevos canales de comunicación y diferentes fuentes de información que benefician en el comportamiento social, los valores y las formas de organización.

Según Pazmiño (2019), el insuficiente uso de la tecnología en la educación y la escasa aplicación de las herramientas innovadoras son causas que influyen en el bajo nivel de aprendizaje, generando conflictos en los procedimientos contextuales, actitudinales, y en la práctica de valores. Es decir, la investigación en didáctica de la química orgánica ha identificado dificultades en sus procesos de enseñanza; se menciona la estructura lógica de los contenidos conceptuales, la exigencia formal y la influencia de los conocimientos y preconcepciones del estudiante

De acuerdo a lo expuesto, se deduce que la insuficiente asimilación de los conceptos de química orgánica, las dificultades en la resolución de ejercicios, y el escaso interés que muestran los estudiantes por aprender o conocer diferentes contenidos, son conflictos que enfrentan los maestros en el ambiente formativo; complejidades que generan problemas de conducta, comportamiento y retrasos en el aprendizaje; los aspectos indicados se reflejan en la falta de entusiasmo en los educandos de tercero de bachillerato de la Unidad Educativa Vicente Anda Aguirre del Cantón Mocha, provincia de Tungurahua, Ecuador, hacía las actividades propuestas en el área con respecto al concepto mencionado.

En el estudio se utilizará la investigación aplicada de diseño experimental, de tipo cuasiexperimental, basadas en el desempeño por funciones básicas, competencias actitudinales, aprendizaje significativo, habilidad cognitiva, competencias procedimentales e interactividad; además, se emplea la investigación básica, pura o fundamental, destinada para resolver los problemas de la sociedad; el trabajo es cuantitativo, la población incluye 32 estudiantes de tercero de bachillerato de la Unidad Educativa Vicente Anda Aguirre del cantón Mocha provincia de Tungurahua, se utiliza la encuesta con 30 preguntas cerradas a través de la escala de Likert, aplica una significación de 0, 9645. 


\subsection{Profundización en el conocimiento}

Durante los últimos años, diversas investigaciones se han enfocado en los procesos de enseñanza y aprendizaje, centrándose en los estudiantes; en este sentido, la aplicación de las Tecnologías de la Información y Comunicación (TIC) aportan en la adquisición del conocimiento de forma sincrónica o asincrónica; por lo expuesto, los investigadores Cruz et al. (2018) manifiestan que la tecnología permite generar ambientes eficaces de aprendizaje que contribuyen en el desarrollo de destrezas, actitudes y habilidades a través de la automatización de las actividades, almacenamiento de datos, e interacción entre computadores y usuarios. En esta perspectiva, las herramientas tecnológicas son indispensables en el proceso de formación que demanda de saberes referentes a la utilización de la información, para dar solución a problemas actuales.

Las tecnologías tienen inicios en los avances científicos de las telecomunicaciones; como mencionan Díaz (2020) y García-Valcárcel (2016), su trascendencia sobresale en el acceso, producción, interacción, tratamiento y comunicación de información, recurso que beneficia en la gestión de materiales de refuerzo para los educandos. Por tanto, la implementación de infraestructura tecnológica promueve el manejo de diferentes software y herramientas digitales; según Cataldi et al. (2011), el laboratorio virtual consolida las investigaciones, experimentos y trabajos de carácter científico o técnico, producido por un sistema informático que surge de la necesidad de apoyar al estudiante en sus prácticas de química.

De la misma manera, Amaya (2009) menciona que los laboratorios virtuales de química orgánica son herramientas digitales que cumplen con la función de fortalecer en el entorno interactivo la atención, concentración y entendimiento para alcanzar el aprendizaje significativo; lamentablemente, en los últimos años han existido diversos escritos que critican a las prácticas de laboratorio en los aspectos metodológicos y conceptuales que engloban conceptos teóricos (Cabero, 2007); sin embargo, no existe duda alguna que los educandos desarrollan su conocimiento mediante la reconstrucción, reflexión, discusión, e interacción con los compañeros, con el profesor, su vivencia y sus intereses.

Desde el punto de vista del constructivismo, la química orgánica es una ciencia teóricoexperimental que activa la habilidad cognitiva de los estudiantes de forma creativa, según Capó (2010), con esta concepción el educando participa en la construcción, reconstrucción de su conocimiento y la toma de decisiones frente a una situación problemática, a diferencia de un ejercicio de tipo automático, beneficia en la argumentación de sus conocimientos y confrontación con la realidad al incluir en sus categorías las funciones básicas, las competencias actitudinales, el aprendizaje significativo, las habilidades cognitivas, las competencias procedimentales, y la interactividad (Mujica, 2016); además, incrementa la motivación al generar actitudes positivas hacia los entornos tecnológicos.

Luego de una revisión de los laboratorios virtuales existentes, a la fecha de la presente investigación, se encuentran algunas opciones que se muestran en la Tabla 1: 
Tabla 1.

Revisión de los laboratorios virtuales

\begin{tabular}{|c|c|c|c|c|c|}
\hline $\begin{array}{l}\text { Nombre de la } \\
\text { aplicación }\end{array}$ & $\begin{array}{l}\text { Adecuación } \\
\text { funcional }\end{array}$ & $\begin{array}{l}\text { Eficiencia en el } \\
\text { rendimiento }\end{array}$ & Utilidad & Confiabilidad & Seguridad \\
\hline \multirow[t]{6}{*}{ YENKA } & $\begin{array}{l}\text { Integridad } \\
\text { funcional }\end{array}$ & $\begin{array}{l}\text { Comportamiento } \\
\text { en el tiempo }\end{array}$ & $\begin{array}{l}\text { Reconocimiento y } \\
\text { conveniencia }\end{array}$ & La madurez & Confidencialidad \\
\hline & $\begin{array}{l}\text { Corrección } \\
\text { funcional }\end{array}$ & $\begin{array}{l}\text { Utilización de } \\
\text { recursos }\end{array}$ & $\begin{array}{l}\text { Capacidad de } \\
\text { aprendizaje }\end{array}$ & Disponibilidad & Integridad \\
\hline & $\begin{array}{l}\text { Idoneidad } \\
\text { funcional }\end{array}$ & Capacidad & Operatividad & Tolerancia a fallos & Aprobación \\
\hline & & & $\begin{array}{l}\text { Protección contra } \\
\text { errores de usuario }\end{array}$ & Recuperación & Responsabilidad \\
\hline & & & $\begin{array}{l}\text { La estética } \\
\text { de interfaz de } \\
\text { usuario }\end{array}$ & & Autenticidad \\
\hline & & & Accesibilidad & & \\
\hline \multirow[t]{6}{*}{$\begin{array}{l}\text { VIRTUAL CHEM } \\
\text { LAB }\end{array}$} & $\begin{array}{l}\text { Integridad } \\
\text { funcional }\end{array}$ & $\begin{array}{l}\text { Comportamiento } \\
\text { en el tiempo }\end{array}$ & $\begin{array}{l}\text { Reconocimiento y } \\
\text { conveniencia }\end{array}$ & La madurez & Confidencialidad \\
\hline & $\begin{array}{l}\text { Corrección } \\
\text { funcional }\end{array}$ & $\begin{array}{l}\text { Utilización de } \\
\text { recursos }\end{array}$ & $\begin{array}{l}\text { Capacidad de } \\
\text { aprendizaje }\end{array}$ & Disponibilidad & Integridad \\
\hline & $\begin{array}{l}\text { Idoneidad } \\
\text { funcional }\end{array}$ & Capacidad & Operatividad & Tolerancia a fallos & Aprobación \\
\hline & & & $\begin{array}{l}\text { Protección contra } \\
\text { errores de usuario }\end{array}$ & Recuperación & Responsabilidad \\
\hline & & & $\begin{array}{l}\text { La estética } \\
\text { de interfaz de } \\
\text { usuario }\end{array}$ & & Autenticidad \\
\hline & & & Accesibilidad & & \\
\hline \multirow{6}{*}{$\begin{array}{l}\text { LABORATORI O } \\
\text { VIRTUAL DE } \\
\text { LA JUNTA } \\
\text { D'ANDALUSIA }\end{array}$} & $\begin{array}{l}\text { Integridad } \\
\text { funcional }\end{array}$ & $\begin{array}{l}\text { Comportamiento } \\
\text { en el tiempo }\end{array}$ & $\begin{array}{l}\text { Reconocimiento y } \\
\text { conveniencia }\end{array}$ & La madurez & Confidencialidad \\
\hline & $\begin{array}{l}\text { Corrección } \\
\text { funcional }\end{array}$ & $\begin{array}{l}\text { Utilización de } \\
\text { recursos }\end{array}$ & $\begin{array}{l}\text { Capacidad de } \\
\text { aprendizaje }\end{array}$ & Disponibilidad & Integridad \\
\hline & $\begin{array}{l}\text { Idoneidad } \\
\text { funcional }\end{array}$ & Capacidad & Operatividad & Tolerancia a fallos & Aprobación \\
\hline & & & $\begin{array}{l}\text { Protección contra } \\
\text { errores de usuario }\end{array}$ & Recuperación & Responsabilidad \\
\hline & & & $\begin{array}{l}\text { La estética } \\
\text { de interfaz de } \\
\text { usuario }\end{array}$ & & Autenticidad \\
\hline & & & Accesibilidad & & \\
\hline
\end{tabular}




\begin{tabular}{|c|c|c|c|c|c|}
\hline \multirow{6}{*}{$\begin{array}{l}\text { BLOG } \\
\text { SALVADOR } \\
\text { HURTADO } \\
\text { FERNÁNDEZ } \\
\text { (LABORATORIO } \\
\text { VIRTUAL) }\end{array}$} & $\begin{array}{l}\text { Integridad } \\
\text { funcional }\end{array}$ & $\begin{array}{l}\text { Comportamiento } \\
\text { en el tiempo }\end{array}$ & $\begin{array}{l}\text { Reconocimiento y } \\
\text { conveniencia }\end{array}$ & La madurez & Confidencialidad \\
\hline & $\begin{array}{l}\text { Corrección } \\
\text { funcional }\end{array}$ & $\begin{array}{l}\text { Utilización de } \\
\text { recursos }\end{array}$ & $\begin{array}{l}\text { Capacidad de } \\
\text { aprendizaje }\end{array}$ & Disponibilidad & Integridad \\
\hline & $\begin{array}{l}\text { Idoneidad } \\
\text { funcional }\end{array}$ & Capacidad & Operatividad & Tolerancia a fallos & Aprobación \\
\hline & & & $\begin{array}{l}\text { Protección contra } \\
\text { errores de usuario }\end{array}$ & Recuperación & Responsabilidad \\
\hline & & & $\begin{array}{l}\text { La estética } \\
\text { de interfaz de } \\
\text { usuario }\end{array}$ & & Autenticidad \\
\hline & & & Accesibilidad & & \\
\hline \multirow[t]{6}{*}{ CHEMLAB } & $\begin{array}{l}\text { Integridad } \\
\text { funcional }\end{array}$ & $\begin{array}{l}\text { Comportamiento } \\
\text { en el tiempo }\end{array}$ & $\begin{array}{l}\text { Reconocimiento y } \\
\text { conveniencia }\end{array}$ & La madurez & Confidencialidad \\
\hline & $\begin{array}{l}\text { Corrección } \\
\text { funcional }\end{array}$ & $\begin{array}{l}\text { Utilización de } \\
\text { recursos }\end{array}$ & $\begin{array}{l}\text { Capacidad de } \\
\text { aprendizaje }\end{array}$ & Disponibilidad & Integridad \\
\hline & $\begin{array}{l}\text { Idoneidad } \\
\text { funcional }\end{array}$ & Capacidad & Operatividad & Tolerancia a fallos & Aprobación \\
\hline & & & $\begin{array}{l}\text { Protección contra } \\
\text { errores de usuario }\end{array}$ & Recuperación & Responsabilidad \\
\hline & & & $\begin{array}{l}\text { La estética } \\
\text { de interfaz de } \\
\text { usuario }\end{array}$ & & Autenticidad \\
\hline & & & Accesibilidad & & \\
\hline \multirow[t]{6}{*}{ PHET } & $\begin{array}{l}\text { Integridad } \\
\text { funcional }\end{array}$ & $\begin{array}{l}\text { Comportamiento } \\
\text { en el tiempo }\end{array}$ & $\begin{array}{l}\text { Reconocimiento y } \\
\text { conveniencia }\end{array}$ & La madurez & Confidencialidad \\
\hline & $\begin{array}{l}\text { Corrección } \\
\text { funcional }\end{array}$ & $\begin{array}{l}\text { Utilización de } \\
\text { recursos }\end{array}$ & $\begin{array}{l}\text { Capacidad de } \\
\text { aprendizaje }\end{array}$ & Disponibilidad & Integridad \\
\hline & $\begin{array}{l}\text { Idoneidad } \\
\text { funcional }\end{array}$ & Capacidad & Operatividad & Tolerancia a fallos & Aprobación \\
\hline & & & $\begin{array}{l}\text { Protección contra } \\
\text { errores de usuario }\end{array}$ & Recuperación & Responsabilidad \\
\hline & & & $\begin{array}{l}\text { La estética } \\
\text { de interfaz de } \\
\text { usuario }\end{array}$ & & Autenticidad \\
\hline & & & Accesibilidad & & \\
\hline
\end{tabular}

Fuente: elaboración propia

En los trabajos de Yánez (2018), Carrión et al. (2020) y Montoya (2012) utilizan el PhET para fortalecer el aprendizaje a través de las prácticas de laboratorio, considerando que forma parte principal en el proceso de la educación y en la formación de estudiantes con pensamiento crítico y constructivistas; obtienen los siguientes resultados:

- Se ajusta a la infraestructura tecnológica, eléctrica y a las situaciones de conectividad.

- Presenta versatilidad y disponibilidad del laboratorio en español y en otros idiomas. 
- En el diseño de actividades de aprendizaje se incluyen instrucciones para desarrollar prácticas de laboratorio.

- Contiene la información de soporte para desarrollar las prácticas.

- Permite efectuar la simulación de una práctica de laboratorio.

- Las actividades diseñadas bajo el título de soluciones son integradas en la plataforma en LMS; pudiendo el educando desarrollar de forma no secuencial.

- Ayuda al mejoramiento del rendimiento académico de una manera positiva.

- Permite que los educandos interactúen en el proceso de enseñanza y aprendizaje de forma innovadora.

Se utilizará el prototipo de integración a plataforma LMS Moodle, empleado por Montoya (2012), que conlleva a la realización de prácticas de laboratorio interactivas, mediante la ubicación de la información y los recursos de cada actividad. Herramienta innovadora que beneficia en la construcción de aulas virtuales, al ofrecer un esquema de trabajo diferente, donde el maestro y los educandos participan de forma activa y dinámica (Rodríguez, 2018).

Martínez y Herriko (2013) mantienen que las principales características LMS son tipos de software libre o propietario, la instalación se realiza en un ordenador personal o servidor; se puede acceder mediante dispositivos móviles y su aplicación se efectúa en función de las necesidades del paradigma educativo.

\section{Metodología}

En el presente apartado se emplea el tipo de estudio propuesto por Fajardo (2018), al cumplir con las condiciones metodológicas de una investigación aplicada de diseño experimental, de tipo cuasi-experimental, por esta razón se incluye en los laboratorios virtuales de química orgánica las funciones básicas, competencias actitudinales, aprendizaje significativo, habilidades cognitivas, competencias procedimentales, e interactividad, componentes que benefician en el balance de ecuaciones, en la construcción de un átomo, en la escala de $\mathrm{pH}$, en los estados de la materia y propiedades de los gases (Cabero, 2007).

En este contexto, se acoge el criterio de los investigadores Ñaupas et al. (2014) al aplicar la indagación básica, pura o fundamental, utilizada para resolver los problemas de la sociedad. Además, de acuerdo con Escobar y García (2019), el trabajo es cuantitativo, de corte cuasiexperimental; además, promueve la comparación de dos equipos en iguales condiciones: el grupo experimental (G.E.), y grupo de control (G.C).

\subsection{Población}

La población involucra a 32 estudiantes de tercero de bachillerato de la Unidad Educativa Vicente Anda Aguirre del cantón Mocha provincia de Tungurahua, son jóvenes entre los 16 y 18 años; una muestra representativa que reúne las características de los sujetos del universo. Se emplea un muestreo no probabilístico o intencionado. 


\subsection{Instrumento}

Por lo expuesto, en el presente estudio se utiliza una encuesta (Tabla 2) conformada por 30 preguntas cerradas dirigidas a los estudiantes; instrumento propuesto por Cobos et al. (2012), utilizado por Montoya (2012); Pérez \& Benavides (2015) y aplicado en la investigación realizada por Escobar y García (2019), incluye las funciones básicas, competencias actitudinales, aprendizaje significativo, habilidad cognitiva, competencias procedimentales, e interactividad, categorías que fueron medidas a través de la escala de Likert con los rangos: no (1), poco (2), indeciso (3), si (4), mucho (5), validado mediante el juicio de tres (3) expertos, aplica una probabilidad $\mathrm{p} \leq 0.05,(5 \%)$, con una confiabilidad del $95 \%$, valor Alpha $=0,968$.

Tabla 2.

Encuesta, competencias en la asignatura de química orgánica

\begin{tabular}{|c|c|}
\hline $\mathrm{N}^{\circ}$. & Ítems \\
\hline \multicolumn{2}{|c|}{ Funciones Básicas } \\
\hline 1 & Me gusta aprender química orgánica con computador. \\
\hline 2 & Me siento a gusto en las clases de química orgánica. \\
\hline 3 & $\begin{array}{l}\text { Aprendería más química orgánica si pudiera usar mayor tiempo en el } \\
\text { computador. }\end{array}$ \\
\hline 4 & Tengo habilidades para el manejo del computador y otras herramientas virtuales. \\
\hline 5 & $\begin{array}{l}\text { El uso de la tecnología empleada por los maestros contribuye en la motivación } \\
\text { por aprender química orgánica. }\end{array}$ \\
\hline \multicolumn{2}{|c|}{ Competencias actitudinales } \\
\hline 6 & $\begin{array}{l}\text { Los recursos tecnológicos utilizados en la clase de química orgánica contribuyen } \\
\text { en la socialización y comunicación. }\end{array}$ \\
\hline 7 & $\begin{array}{l}\text { Las herramientas tecnológicas empleadas por el docente contribuye con la } \\
\text { autonomía y la gestión del tiempo en la clase de química orgánica. }\end{array}$ \\
\hline 8 & $\begin{array}{l}\text { Me veo apoyado(a) por las herramientas de Internet en el aprendizaje de la } \\
\text { química orgánica. }\end{array}$ \\
\hline 9 & Puedo resolver las actividades planteadas en un laboratorio virtual. \\
\hline 10 & El laboratorio virtual facilita en el aprendizaje de química orgánica. \\
\hline \multicolumn{2}{|c|}{ Aprendizaje Significativo } \\
\hline 11 & Tengo dificultades en el aprendizaje de química orgánica. \\
\hline 12 & Los conceptos que se estudian en química orgánica son difíciles de aprender. \\
\hline 13 & $\begin{array}{l}\text { Las clases de química orgánica me agradan porque encuentro relación con la } \\
\text { vida cotidiana. }\end{array}$ \\
\hline 14 & $\begin{array}{l}\text { El uso de la tecnología por los docentes contribuye en los estudiantes para } \\
\text { recordar, comprender, aplicar y analizar los temas tratados en química orgánica. }\end{array}$ \\
\hline 15 & $\begin{array}{l}\text { La utilización de recursos tecnológicos por los docentes contribuye para } \\
\text { aprender en base a: estudios de casos, laboratorios y proyectos relacionados } \\
\text { con la asignatura. }\end{array}$ \\
\hline \multicolumn{2}{|c|}{ Habilidad cognitiva } \\
\hline 16 & $\begin{array}{l}\text { El empleo de herramientas digitales por los maestros contribuye en la resolución } \\
\text { de problemas }(\mathrm{ABP}) \text { con creatividad referente a los temas tratados en química } \\
\text { orgánica. }\end{array}$ \\
\hline
\end{tabular}




\begin{tabular}{|c|c|}
\hline 17 & $\begin{array}{l}\text { La realización de prácticas de laboratorio virtual ayuda al aprendizaje de } \\
\text { química orgánica. }\end{array}$ \\
\hline 18 & $\begin{array}{l}\text { Lo que aprendo en química orgánica me permite explicar lo observado en las } \\
\text { prácticas de laboratorio virtual. }\end{array}$ \\
\hline 19 & $\begin{array}{l}\text { Me gusta realizar experimentos de química orgánica porque aprendo a } \\
\text { investigar. }\end{array}$ \\
\hline 20 & $\begin{array}{l}\text { Conozco algunos de los procedimientos de trabajo que se realizan en un } \\
\text { laboratorio virtual de química orgánica. }\end{array}$ \\
\hline \multicolumn{2}{|c|}{ Competencias procedimentales } \\
\hline 21 & $\begin{array}{l}\text { El manejo de las aplicaciones de Microsoft Office por los maestros apoya para } \\
\text { el aprendizaje de la asignatura. }\end{array}$ \\
\hline 22 & $\begin{array}{l}\text { El uso de herramientas virtuales por los maestros, contribuye para el } \\
\text { aprendizaje de química orgánica. }\end{array}$ \\
\hline 23 & $\begin{array}{l}\text { La utilización de redes sociales por los docentes, contribuye para el aprendizaje } \\
\text { de química orgánica. }\end{array}$ \\
\hline 24 & $\begin{array}{l}\text { El uso de presentaciones con texto, imágenes, videos con actividades digitales } \\
\text { interactivos por los docentes; contribuyen con el aprendizaje de química } \\
\text { orgánica. }\end{array}$ \\
\hline 25 & $\begin{array}{l}\text { El uso de presentaciones con texto, imágenes, videos y reflexión por los } \\
\text { docentes; contribuyen con el aprendizaje de química orgánica. }\end{array}$ \\
\hline \multicolumn{2}{|c|}{ Interactividad } \\
\hline 26 & $\begin{array}{l}\text { Permitir el uso de las TIC a los estudiantes (Laptop, tabletas, cámaras, etc.), por } \\
\text { los docentes; contribuyen con el aprendizaje de química orgánica. }\end{array}$ \\
\hline 27 & $\begin{array}{l}\text { El uso de tablas editadas, imágenes, ecuaciones (tamaño, ajuste con el texto, } \\
\text { etc.) por los docentes; contribuyen con el aprendizaje de química orgánica. }\end{array}$ \\
\hline 28 & $\begin{array}{l}\text { Empleo mis habilidades en el uso de Internet y las herramientas virtuales para } \\
\text { aprender química orgánica. }\end{array}$ \\
\hline 29 & $\begin{array}{l}\text { Me gustan las clases que utilizan laboratorios virtuales para enseñar y aprender } \\
\text { química orgánica. }\end{array}$ \\
\hline 30 & Los laboratorios virtuales ayudan al aprendizaje de química orgánica \\
\hline
\end{tabular}

Fuente: elaboración propia

La aprobación del instrumento se realizó a través del Coeficiente Alfa de Cronbach, como se evidencia en la Tabla 3, con una comprobación de los ítems, evaluando en el 0,968 recopila información fiable, considerándose las mediciones sólidas y consistentes, Villanueva (2016) afirma que el valor de fiabilidad para la investigación básica es entre 0.7 y 0.8 ; en el estudio aplicado equivale a 0.99 , es excelente, mientras el coeficiente 0,8 es bueno.

\section{Resultados}

\subsection{Análisis}

Antes y después de utilizar el simulador virtual, como herramienta para la enseñanza de química orgánica, con base en los resultados obtenidos en la encuesta dirigida a los estudiantes de tercero de bachillerato de la Unidad Educativa Vicente Anda Aguirre, del grupo de control y experimental, se obtienen los siguientes resultados: 
El análisis efectuado en el pre test relacionado con las funciones básicas indicó que los estudiantes, en un $25 \%$, no aprenden química orgánica con la computadora, presentan dificultades en el manejo de aplicaciones informáticas. En las competencias actitudinales, el $28 \%$ de docentes no utilizan herramientas tecnológicas; sin embargo, un 19\% se apoyan en el Internet para recabar información; al 31\% no le agradan las clases magistrales; en el post test, se induce que el $100 \%$ adquiere el aprendizaje significativo mediante la práctica y la socialización, el 100\% considera que reforzó el conocimiento y entendimiento en la asignatura.

Los resultados indicaron en el pre test de las competencias cognitivas que en un $25 \%$, las herramientas digitales empleadas por los maestros no contribuyen en la resolución de problemas (ABP), el $31 \%$ ocasionalmente realiza prácticas de laboratorio virtual, el $31 \%$ no efectúa experimentos, no investiga. En referencia a las competencias procedimentales, el $15 \%$ no aprovecha las aplicaciones de Microsoft Office, el $31 \%$ a veces utiliza redes sociales, el $25 \%$ no construye presentaciones con texto, imágenes, videos. En cuanto a interactividad, el $28 \%$ no emplea laptop, tabletas, cámaras, entre otros, el $34 \%$ en ocasiones utilizan laboratorios virtuales para enseñar y aprender química orgánica. En el post test se supera el $100 \%$ de los estudiantes evaluados y se obtiene un desempeño superior. 
Tabla 3.

Correlaciones

\begin{tabular}{|c|c|c|c|c|c|c|c|c|}
\hline & & & $\begin{array}{c}\text { D1 } \\
\text { Funciones } \\
\text { Básicas }\end{array}$ & $\begin{array}{c}\text { D2 } \\
\text { Competen- } \\
\text { cias Actitudi- } \\
\text { nales }\end{array}$ & $\begin{array}{c}\text { D3 } \\
\text { Aprendizaje } \\
\text { Significativo }\end{array}$ & $\begin{array}{c}\text { D4 } \\
\text { Habilidad } \\
\text { Cognitiva }\end{array}$ & \begin{tabular}{|c} 
D5 \\
Competen- \\
cias \\
$\begin{array}{c}\text { Procedimen- } \\
\text { tal. }\end{array}$ \\
\end{tabular} & $\begin{array}{c}\text { D6 } \\
\text { Interactividad }\end{array}$ \\
\hline \multirow{18}{*}{$\begin{array}{l}\text { Rho de } \\
\text { Spearman }\end{array}$} & \multirow{3}{*}{$\begin{array}{l}\text { D1 } \\
\text { Funciones } \\
\text { Básicas }\end{array}$} & $\begin{array}{l}\text { Coeficiente } \\
\text { de correlación }\end{array}$ & 1.000 & $.801^{* *}$ & $.668^{* *}$ & $.501^{* *}$ & $.625^{* *}$ & $.381^{*}$ \\
\hline & & Sig. (bilateral) & . & .000 & .000 & .003 & .000 & .031 \\
\hline & & N & 32 & 32 & 32 & 32 & 32 & 32 \\
\hline & \multirow{3}{*}{$\begin{array}{l}\text { D2 } \\
\text { Competen- } \\
\text { cias Actitudi- } \\
\text { nales }\end{array}$} & $\begin{array}{l}\text { Coeficiente } \\
\text { de correlación }\end{array}$ & $.801^{* *}$ & 1.000 & $.690^{* *}$ & $.479^{* *}$ & $.596^{\prime \prime}$ & $.400^{*}$ \\
\hline & & Sig. (bilateral) & .000 & . & .000 & .006 & .000 & .023 \\
\hline & & $N$ & 32 & 32 & 32 & 32 & 32 & 32 \\
\hline & \multirow{3}{*}{$\begin{array}{l}\text { D3 } \\
\text { Aprendizaje } \\
\text { Significativo }\end{array}$} & $\begin{array}{l}\text { Coeficiente } \\
\text { de correlación }\end{array}$ & $.668^{*}$ & $.690^{* *}$ & 1.000 & $.683^{* \prime}$ & $.596^{\prime \prime}$ & $.400^{*}$ \\
\hline & & Sig. (bilateral) & .000 & .000 & . & .000 & .000 & .023 \\
\hline & & $\mathrm{N}$ & 32 & 32 & 32 & 32 & 32 & 32 \\
\hline & \multirow{3}{*}{\begin{tabular}{|l} 
D4 \\
Habilidad \\
Cognitiva
\end{tabular}} & $\begin{array}{l}\text { Coeficiente } \\
\text { de correlación }\end{array}$ & $.501^{* *}$ & $.479^{* *}$ & $.683^{* \prime}$ & 1.000 & $.861^{\prime \prime}$ & $.525^{\prime \prime}$ \\
\hline & & Sig. (bilateral) & .003 & .006 & .000 & & .000 & .002 \\
\hline & & N & 32 & 32 & 32 & 32 & 32 & 32 \\
\hline & \multirow{3}{*}{$\begin{array}{l}\text { D5 } \\
\text { Competen- } \\
\text { cias } \\
\text { Procedimen- } \\
\text { tal. }\end{array}$} & \begin{tabular}{|l|} 
Coeficiente \\
de correlación
\end{tabular} & $.625^{* *}$ & $.596^{* *}$ & $.596^{* *}$ & $.861^{\prime \prime}$ & 1.000 & $.596^{\prime \prime}$ \\
\hline & & Sig. (bilateral) & .000 & .000 & .000 & .000 & & .000 \\
\hline & & $N$ & 32 & 32 & 32 & 32 & 32 & 32 \\
\hline & \multirow{3}{*}{$\begin{array}{l}\text { D6 Interacti- } \\
\text { vidad }\end{array}$} & $\begin{array}{l}\text { Coeficiente } \\
\text { de correlación }\end{array}$ & $.381^{*}$ & $.400^{*}$ & $.400^{\circ}$ & $.525^{* *}$ & $.596^{* *}$ & 1.000 \\
\hline & & Sig. (bilateral) & .031 & .023 & .023 & .002 & .000 & \\
\hline & & $N$ & 32 & 32 & 32 & 32 & 32 & 32 \\
\hline
\end{tabular}

Fuente: elaboración propia

Con una población de 32 estudiantes, se efectúa la correlación entre el pre test y el post test; así, en el muestreo de error simple, el intervalo de confianza es 95\%, una significación de 0,01 y 0,05 no existen valores negativos, por tanto, la correlación de Rho de Spearman $(0,801$ y 5,96) es mayor a cero, lo que significa que es positiva y perfecta, existiendo una asociación lineal moderada. 
El estudio se efectuó en un periodo de 5 meses (noviembre 2020 - abril 2021) con estudiantes de química orgánica de tercer año de bachillerato general unificado, en el laboratorio virtual las unidades del programa de aprendizaje incluidas en cinco talleres incluyeron:

1. Balance de ecuaciones químicas

2. Construye un átomo

3. Escala de $\mathrm{PH}$

4. Estados de la materia

5. Propiedades de los gases

Entre las herramientas y recursos tecnológicos utilizados sobresalen los siguientes:

Los videos guía orientaron al estudiante en el acceso a los simuladores del laboratorio virtual PHET; para el efecto se utiliza una página web, la conexión de Internet, un computador, Tablet o Smartphone, un usuario y la contraseña entregada por la docente.

El material didáctico permitió visualizar los conceptos teóricos abordados durante el curso de química orgánica que incluye: 23 imágenes a color y seis guías en PDF, para el efecto se utiliza la herramienta FLIPHTML5; además se agregan cuatro videos desde Google Drive; cinco simuladores PHET y seis evaluaciones. Se resalta que los recursos fueron seleccionados de forma organizada, metódica y sistemática, con la finalidad de innovar y alcanzar la interacción en el proceso educativo, al mismo tiempo, apoyar a los educandos en su papel de constructor de su propio conocimiento.

Otro criterio significativo para la didáctica fue la selección de los simuladores que facilitó el ingreso de los educandos, la finalidad fue retroalimentar los conocimientos, mejorar el rendimiento y desempeño académico mediante las prácticas que incluyeron representaciones, y descripciones referentes a las unidades: ecuaciones químicas, conservación de la masa, particularidades de los átomos, estructura atómica, símbolos isotópicos, núcleo atómico, generalidades del $\mathrm{PH}$, ácidos, bases, dilución, describir las características de los tres estados de la materia (sólido, líquido y gaseoso) y explicar la congelación y la fusión con detalle a nivel molecular, la concentración, la temperatura, y el radio afectan la tasa de difusión.

Con el fin de explorar las actividades como estrategia de aprendizaje, se incluyeron simuladores, utilizando los programas PHET, elaborados en un tiempo de cuatro semanas, cada uno incluye cuarenta minutos destinados a la exposición durante la clase; los temas asignados fueron impartidos previamente por la docente. La dinámica consistió en proyectar en la pantalla, considerando que los simuladores en su mayoría se utilizaron fuera del aula, por tal razón se efectuó el registro en un formato electrónico.

\subsection{Forma de presentación del laboratorio virtual}

A continuación, en las siguientes figuras $1,2,3,4,5,6,7,8,9$ y 10 se presenta el curso creado en la página https://fernandaarroba.com/my 


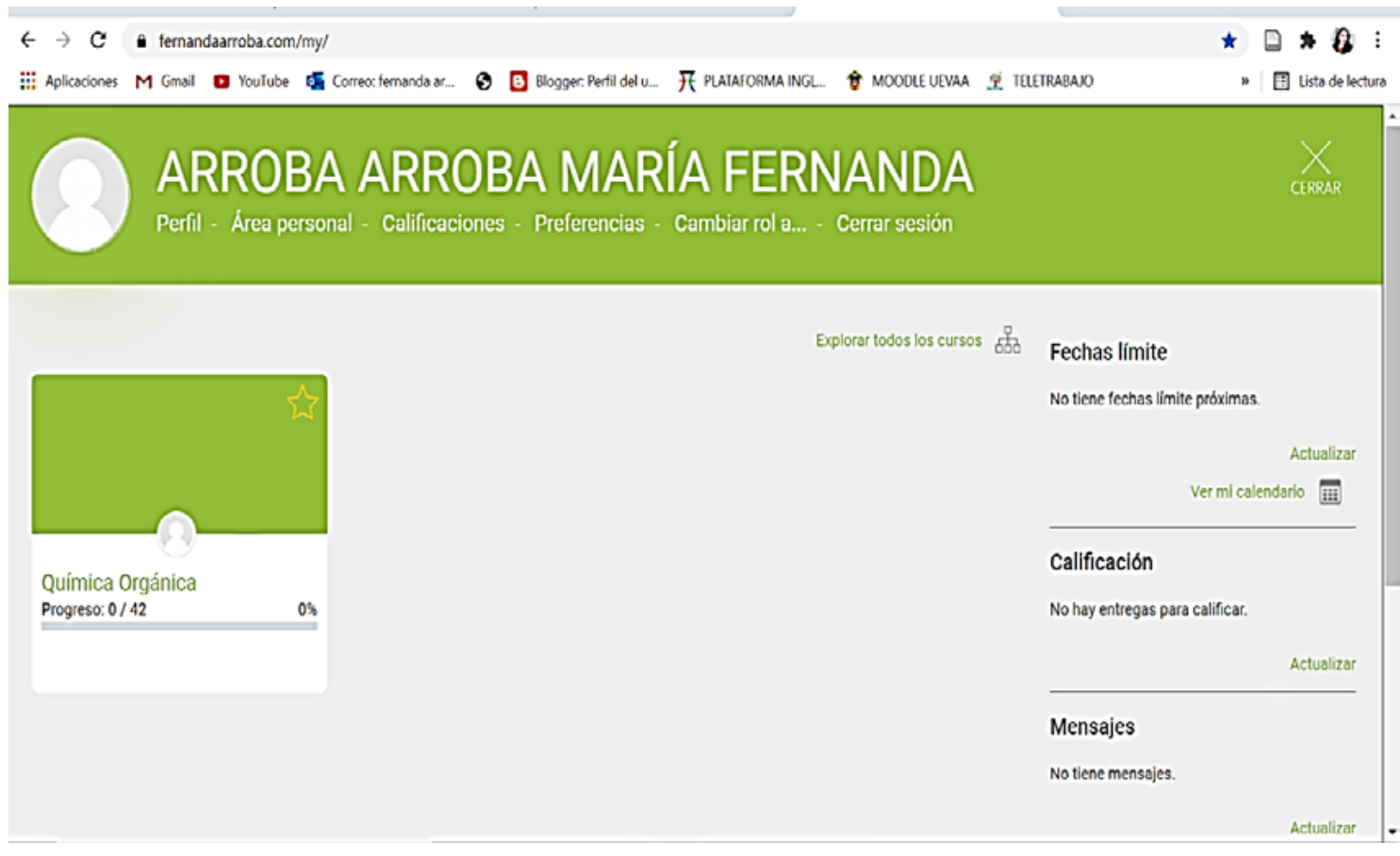

Figura 1. Inicio del curso

Fuente: elaboración propia

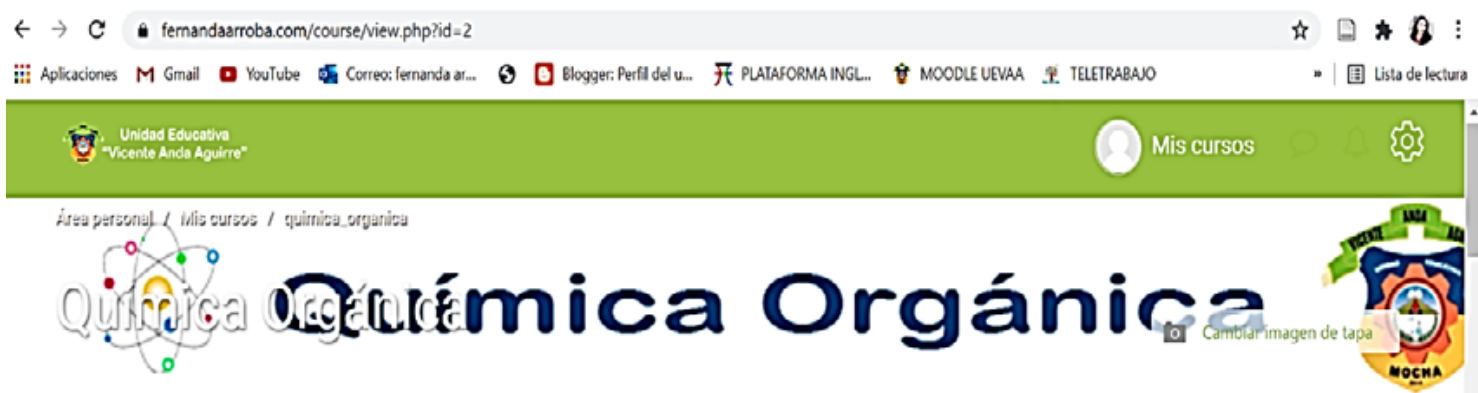

CONTENIDO

Q. Presentación

Presentación

Progreso: $0 / 3$

Introducción

Practica 1

Progreso: $0 / 6$

Practica 2

Progresa: $0 / 8$

Practica 3

¡Bienvenidos al curso.
MOOC QUÍMICA ORGÁNICA

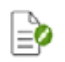

Figura 2. Contenidos del curso de química orgánica

Fuente: elaboración propia 


\section{Introducción sobre el Curso de Química Orgánica}

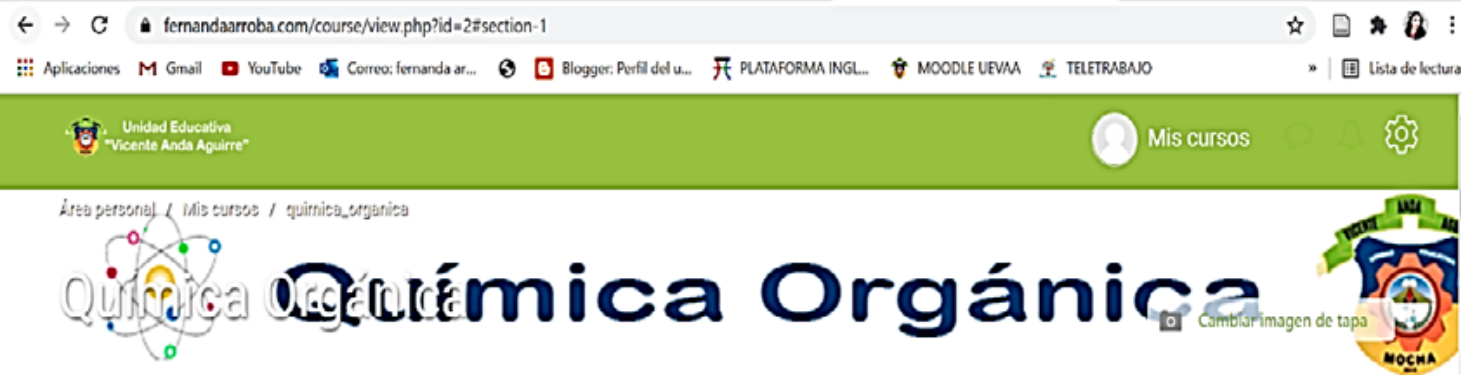

CONTENIDO Q. Introducción

Presentación

Progresa $0 / 3$

Conoce más de la Química Orgánica

Introducción

Practica 1

Progreso: 0/6

Practica 2

Progreso: 0/8

Instrucciones:

1. Haga clic en cada una de las secciones del panel izquierdo y navegue entre los módulos de estudio del presente curso de Introducción a la Vida Universitaria.

Figura 3. Introducción del curso de química orgánica

Fuente: elaboración propia

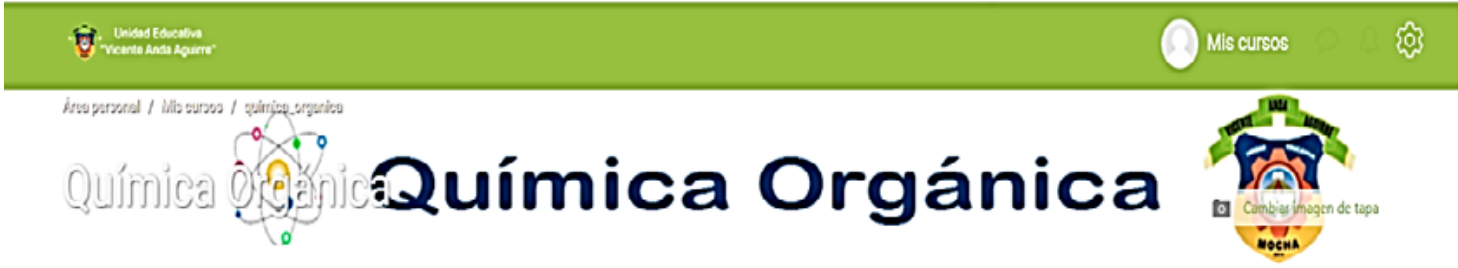

\begin{tabular}{|c|}
\hline CONTENIDO \\
\hline $\begin{array}{l}\text { Presentación } \\
\text { Progreso } 0 / 3\end{array}$ \\
\hline Introducción \\
\hline $\begin{array}{l}\text { Practica } 1 \\
\text { Progess } 0 / 6\end{array}$ \\
\hline $\begin{array}{l}\text { Practica } 2 \\
\text { Progrest } 0 / 8\end{array}$ \\
\hline $\begin{array}{l}\text { Practica } 3 \\
\text { Progess } 0 / 8\end{array}$ \\
\hline $\begin{array}{l}\text { Practica } 4 \\
\text { Proutsu } 0 / 8\end{array}$ \\
\hline $\begin{array}{l}\text { Practica } 5 \\
\text { Propresa 0/7 }\end{array}$ \\
\hline
\end{tabular}
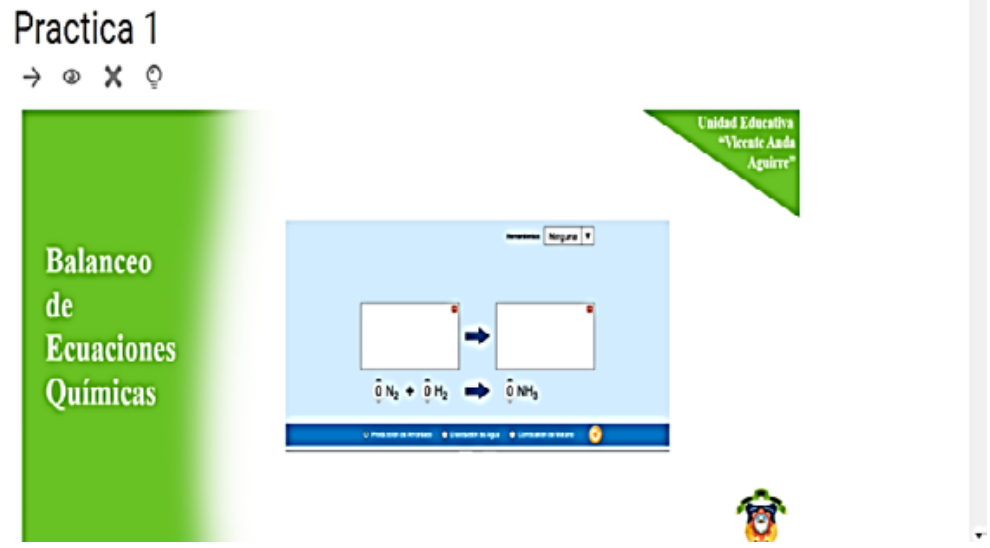

Figura 4. Simulador de la práctica \#1 balance de ecuaciones

Fuente: elaboración propia 


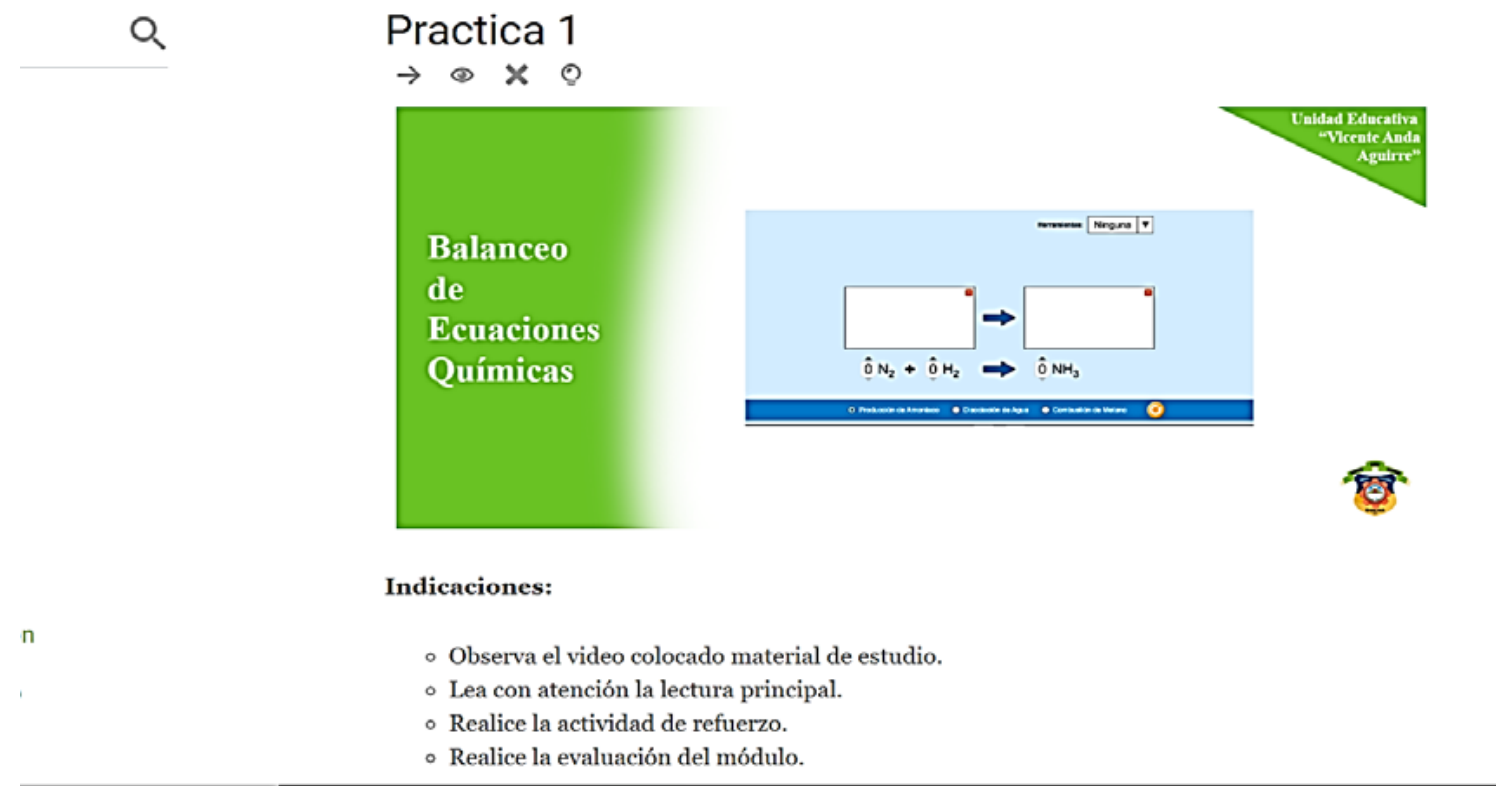

Figura 5. Indicaciones de la práctica \#1 balance de ecuaciones

Fuente: elaboración propia

\section{MLATIEIRIAVL DE ESTUUDIO}

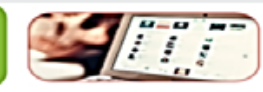

$\rightarrow$

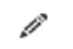

:

\section{OBJETIVOS}

Al utilizar este simulador y realizar los ejercicios propuestos, el estudiante será capaz de:

1. Interpretar el significado de una ecuación química, lo que implica:

a) distinguir productos y reactivos

b) visualizar el significado de los subíndices en las fórmulas.

c) distinguir átomos y moléculas. d) utilizar correctamente los coeficientes estequiométricos en las ecuaciones químicas.

2. Verificar la ley de la conservación de la materia en una reacción química por medio del balance de la ecuación química correspondiente.

\section{INTRODUCCIÓN}

Figura 6. Objetivo de la práctica \#1 balance de ecuaciones

Fuente: elaboración propia 
$\leftarrow \rightarrow \mathrm{C}$ fernandaarroba.com/course/view.php?id=2" section-2

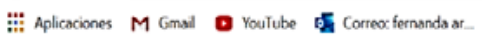

(3) Blogger Perfil del

F PLAIAFORMA INGL

tิ

modole ueva si teletrabajo

$Q$ \& $\triangle * B$ :

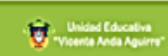

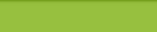

(n)

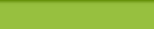

(a)

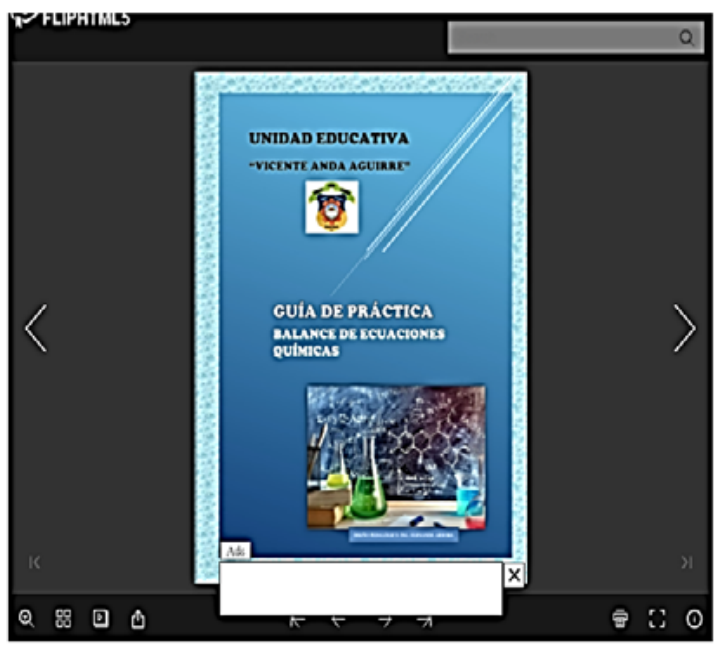

Figura 7. Guía práctica \#1 balance de ecuaciones

Fuente: elaboración propia

- fernandaarroba.com/course/view.php? id=2\#section-2

M Gmail D YouTube is Correo: fernanda ar
3 .
Q म $\mathrm{A} \Rightarrow \boldsymbol{B}$ : " 国 Lista de lectura

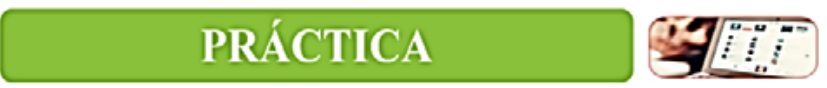

$\rightarrow$

$\vdots$

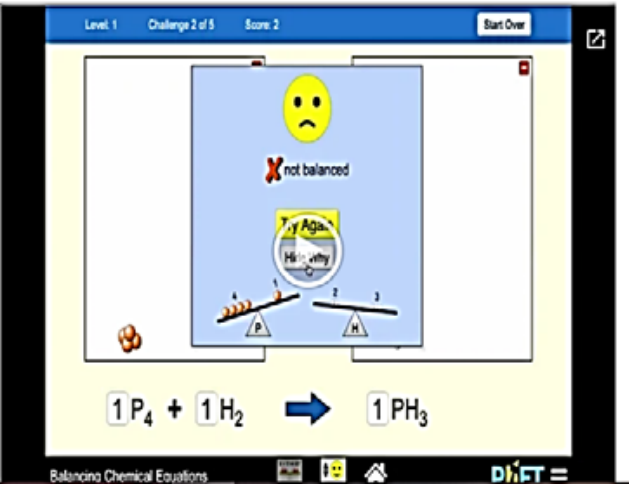

Figura 8. Video tutorial para la simulación práctica \#1 balance de ecuaciones

Fuente: elaboración propia 


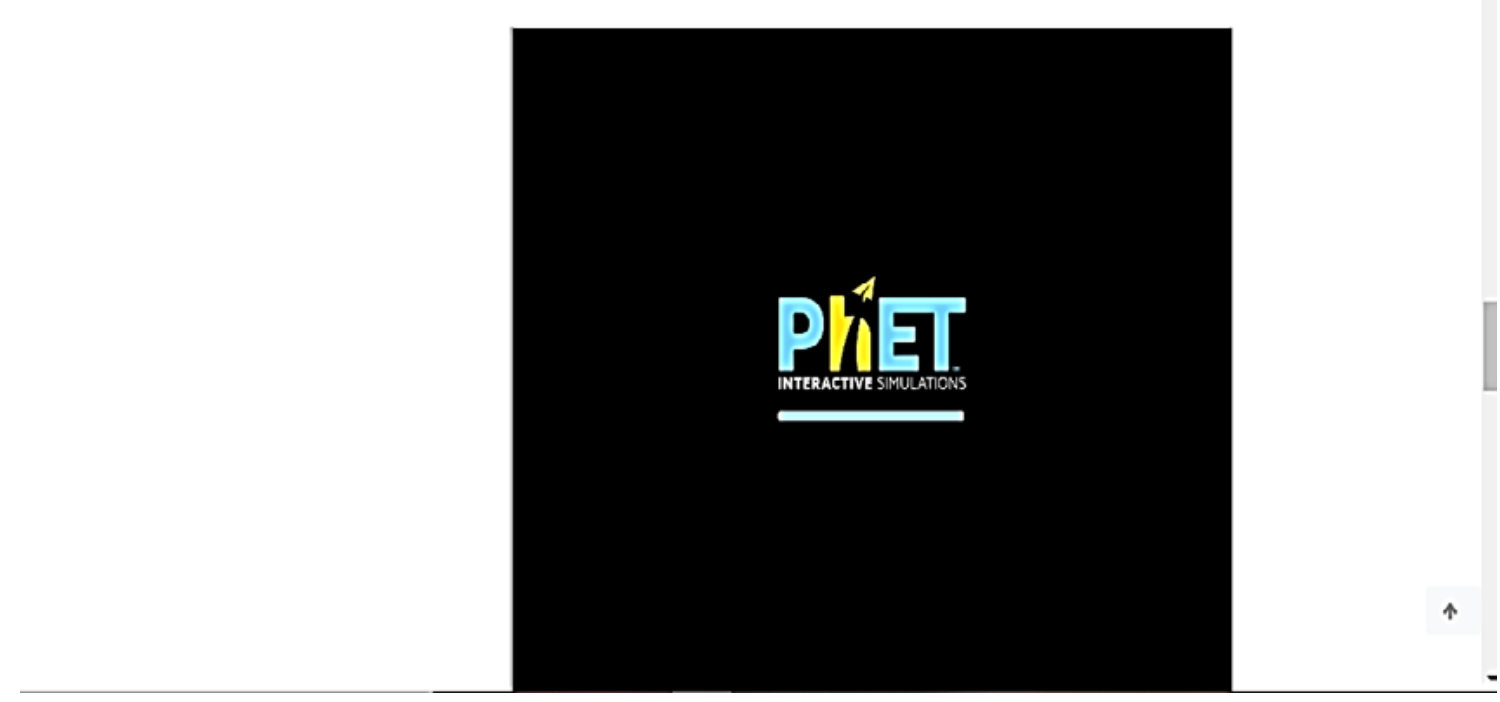

Figura 9. Simulador del laboratorio virtual PHET Fuente: elaboración propia
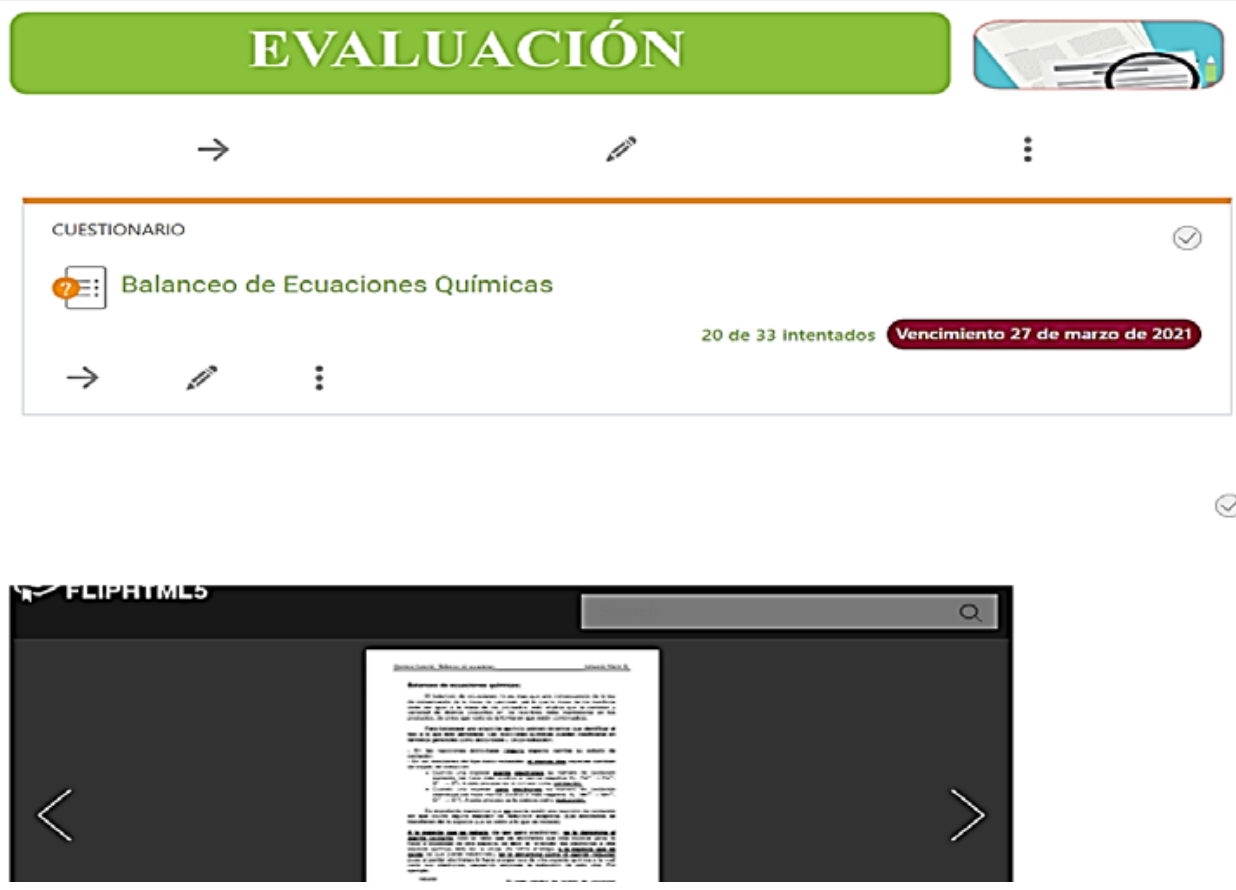

Figura 10. Evaluación de la práctica \#1 balance de ecuaciones

Fuente: elaboración propia 


\subsection{Deducciones}

Para iniciar la investigación se revisó los diferentes proveedores de hosting (Hostinger, HostGator y Hosting Ecuador), seleccionando Hosting Ecuador, considerando la cuenta que posee para pequeñas empresas (espacio de $3.000 \mathrm{MB}$, transferencia mensual de $10.000 \mathrm{MB}$ ); de forma adicional se realizó un incremento de $150 \mathrm{~GB}$; LMS con un alto tráfico de datos; por esta razón, el plan inicial no abastecía en las evaluaciones de los estudiantes; además, se adquirió un dominio que permitió visualizar la página https://fernandaarroba.com/

Posteriormente se analizó la información relacionada con los LMS disponibles (Moodle, Chamilo, Canvas) optando por la plataforma Moodle 3.10 última versión estable, liberada para uso gratuito. Para la utilización del aula virtual se creó una guía instruccional que referencia los recursos y su ubicación.

\section{Conclusiones}

Según los resultados obtenidos en el uso del laboratorio virtual de química orgánica, aplicado en la Unidad Educativa Vicente Anda Aguirre, se obtienen las siguientes conclusiones:

La utilización de las Tecnologías de la Información y Comunicación facilitan el proceso de enseñanza a través de herramientas virtuales que benefician en la realización de experimentos, con una elevada aproximación a la realidad, de esta forma facilita al estudiante la relación del conocimiento adquirido mediante la teoría, las clases magistrales y la práctica, obteniendo como resultado el aprendizaje significativo.

Se considera apropiado emplear laboratorios virtuales con simuladores que refuercen las funciones básicas (atención, concentración y entendimiento), las competencias actitudinales (comportamiento), el aprendizaje significativo (integración de la teoría y la práctica), habilidad cognitiva (toma de decisiones, solución de problemas), competencias procedimentales (pensamiento y reflexión), interactividad (procesos de detección, selección, organización y uso de la información).

El laboratorio virtual se ha constituido en una herramienta pedagógica tecnológica en la enseñanza de química orgánica, potencializa la utilización de la red e Internet y mejora la ejecución de prácticas, por lo tanto, la tecnología beneficia en el trabajo experimental, el rendimiento académico, la experiencia y el aprendizaje significativo.

\section{Referencias}

Amaya, F. (2009). Laboratorios reales versus laboratorios virtuales, en la enseñanza de la física. El Hombre y la Máquina, (33) 82-95. https://www.redalyc.org/pdf/478/47812225009.pdf

Bermeo, M., Peralta, I., Remache, W., \& Mayorga, E. (09 de marzo de 2018). Índice de repitencia y sus causas de la Facultad de Ciencias Químicas de la Universidad Central del Ecuador. Revista CIEG, (31), 109-127. 
Cabero, J. (2007). Las TIC en la enseñanza de la química: aportaciones desde la Tecnología Educativa. In Bodalo, A. y otros (editores). Química: vida y progreso. Asociación de químicos de Murcia.

Capó, J. (2010). Docencia de asignatura de gestión en una ingeniería. Utilización de metodologías activas de aprendizaje. Revista de Formación e Innovación Educativa Universitaria, 3(2) 97-111.

Carrión, F., García, D., Erazo, C., \& Erazo, J. (2020). Simulador virtual PhET como estrategia metodológica para el aprendizaje de Química. CIENCIAMATRIA, 6(3), 193-216. https://doi.org/10.35381/cm.v6i3.396

Cataldi, Z., Chiarenza, D., Dominighini, C., \& Lage, F. (2011). Enseñando Química con TICs: Propuesta de Evaluación Laboratorios Virtuales de Química (LVQs). In XIV Congreso Internacional EDUTEC, 26.

Cobos, D., López, E., Jaén, A., Martín, A., \& Molina, L. (2012). Actas del Congreso. I Congreso Internacional Virtual Innovagogía 2012. Congreso Virtual sobre innovación pedagógica y praxis educativa. AFOE Asociación para la Formación, el Ocio y el Empleo.

Cruz, M., Pozo, M., Andino, A., \& Arias, A. (2018). Las tecnologías de la información y la comunicación (TIC) como forma investigativa interdisciplinaria con un enfoque intercultural para el proceso de formación de los estudiantes. Revista científica electrónica de Educación y Comunicación en la Sociedad del Conocimiento, 18(2), 196-215 https://doi.org/10.30827/eticanet.v18i2.11889

Díaz, N. (2020). Aula virtual semántica para reforzar el aprendizaje de Procesador de texto en estudiantes de octavo año de Educación General Básica. Universidad Tecnológica Israel.

Escobar, O., \& García, C. (2019). Uso didáctico del laboratorio virtual y su influencia en el aprendizaje de las unidades químicas de masa por competencias en estudiantes de los grados 10 y 11 en la institución educativa Fe y Alegría Aures de Medellín, 2015. [Tesis de Posgrado, Universidad Privada Norbert Wiener] Repositorio UWIENER http://repositorio.uwiener.edu.pe/handle/123456789/3520

Fajardo, E. (2018). Filosofía y ciencia: fuente y generación de método y conocimiento verdadero. Amauta, 16(31), 9-32.

García-Valcárcel, A. (2016). Recursos digitales para la mejora de la enseñanza y el aprendizaje. Universidad de Salamanca. Repositorio Documental Gredos. http://hdl.handle.net/10366/131421

Martínez, P., \& Herriko, E. (2013). Los LMS como herramienta colaborativa en educación. In presentado en V Congreso Internacional Latina de Comunicación Social (V CILCS), Universidad de La Laguna.

Montoya, D. (2012). Diseño e implementación de guías para el aprendizaje de la materia y sus propiedades apoyadas en herramientas virtuales. [Tesis de grado, Universidad Nacional de Colombia]. Repositorio UNAL https://repositorio.unal.edu.co/handle/unal/11631

Mujica, R. (2016). La tecnología en la educación. Revista Tecnológica Educativa Docente, 4(4). https://ojs. docentes20.com/index.php/revista-docentes20/article/view/57 
Ñaupas, H., Novoa, E., Mejía, E., \& y Villagómez, A. (2014). Metodología de la investigación cuantitativacualitativa y redacción de la tesis (Cuarta ed.). Ediciones de la U.

Pazmiño, M. (2019). Influencia del estilo de aprendizaje en el rendimiento académico de los estudiantes en la asignatura química orgánica. Universidad de Guayaquil.

Pérez, H., \& Benavides, L. (2015). Objetos virtuales de aprendizaje y un laboratorio virtual de química en la enseñanza de la ley de conservación de la masa. Historia de la Educación Colombiana, 18(18), 169-200. https://doi.org/10.22267/rhec.151818.34

Rodríguez, Y. T. (2018). Entorno virtual de aprendizaje centrado en la metáfora educativa. Universidad Ciencia y Tecnología, 22(86), 11-11.

Sepúlveda, L. (2014). La incorporación de la tecnología en la enseñanza de la química. [Tesis doctoral, Universidad del Valle]. Biblioteca Digital UNIVALLE http://hdl.handle.net/10893/7189

Villanueva, S. (2016). Alfa Cronbach, Prueba de regresión lineal, Prueba T, Chi cuadrada \& Coeficiente de correlación de Pearson. Pedagogía, 12.

Yánez, A. (2018). Simulador PhET en la enseñanza de las cargas eléctricas en movimiento en los estudiantes de tercero de bachillerato de la Unidad Educativa "Paúl Dirac", durante el año lectivo 20172018. [Tesis de grado, Universidad Central del Ecuador]. Repositorio UCE. http://www.dspace.uce.edu.ec/ handle/25000/15336 
Copyright (c) 2021 María Fernanda Arroba Arroba y Santiago Alejandro Acurio Maldonado

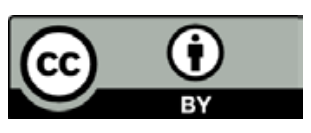

Este texto está protegido bajo una licencia internacional Creative Commons 4.0.

Usted es libre para Compartir-copiar y redistribuir el material en cualquier medio o formato

- y Adaptar el documento - remezclar, transformar y crear a partir del material-para cualquier propósito, incluso para fines comerciales, siempre que cumpla las condiciones de Atribución. Usted debe dar crédito a la obra original de manera adecuada, proporcionar un enlace a la licencia, e indicar si se han realizado cambios. Puede hacerlo en cualquier forma razonable, pero no de forma tal que sugiera que tiene el apoyo del licenciante o lo recibe por el uso que hace de la obra.

\section{$\underline{\text { Resumen de licencia - Texto completo de la licencia }}$}

